\title{
Engineering model for low-velocity impacts of multi-material cylinder on a rigid boundary
}

\author{
M.F. Buchely ${ }^{1}$, A. Maranon ${ }^{1, a}$, and F. Delvare ${ }^{2}$ \\ 1 Structural Integrity Research group, Department of Mechanical Engineering, Universidad de los Andes, Cr 1 \\ Este 19A-40 Bogota, Colombia \\ 2 Institut PRISME, ENSI de Bourges 88 boulevard Lahitolle, 18020 Bourges Cedex, France
}

\begin{abstract}
Modern ballistic problems involve the impact of multi-material projectiles. In order to model the impact phenomenon, different levels of analysis can be developed: empirical, engineering and simulation models. Engineering models are important because they allow the understanding of the physical phenomenon of the impact materials. However, some simplifications can be assumed to reduce the number of variables. For example, some engineering models have been developed to approximate the behavior of single cylinders when impacts a rigid surface. However, the cylinder deformation depends of its instantaneous velocity. At this work, an analytical model is proposed for modeling the behavior of a unique cylinder composed of two different metals cylinders over a rigid surface. Material models are assumed as rigid-perfectly plastic. Differential equation systems are solved using a numerical Runge-Kutta method. Results are compared with computational simulations using AUTODYN 2D hydrocode. It was found a good agreement between engineering model and simulation results. Model is limited by the impact velocity which is transition at the interface point given by the hydro dynamical pressure proposed by Tate.
\end{abstract}

\section{Introduction}

The evolution of armor systems has led the development of different types of mult-material projectiles [1]. Some projectiles are composed by a hard nose and a heavy body in order to increase the damage over the armor [2], where the most common materials used are: lead and aluminum (as body) and steel and tungsten (as a hard nose) [1].

The material response of projectile is complex; therefore, many simplifications of the impact system have been assumed for constructing simple engineering analytical models. Thus, the number of variables is reduced and the physical phenomenon can be understood inside the materials. For example, it is common to assume a deformable projectile impacting a rigid wall. Taylor [3] was the first person who used this configuration to study projectile material behavior.

According to Taylor; when a solid cylinder impacts a rigid wall, it suffers elastic and plastic stress inside the projectile moving as waves. Cylinder deceleration is due the movements and interactions of waves [3,4]. Taylor reduced this complex behavior to one dimensional problem, where the radial effects are neglected and dynamical strength stress and density can be assumed constants. Lee-Tupper [5] developed a model involving the wave movements inside the projectile and the material strain hardening. Howkyard [6] developed a model using the energy conservation equations over the projectile. Jones [7-9] proposed a three stages model involving new variables as particle velocity, strain hardening, area changes. Jones model produce good approximations over a wide velocity range; although, it depends of some variables that can be experimentally measured.

Previous models do not show a good agreement for high impact velocities. For this reason, White [10]

a e-mail: emaranon@uniandes.edu.co separated the deformation of cylinders in two modes: Mode I, as a mushroom deformation for low impact velocities (the cylinder is deformed but remains coherent); and Mode II, for high velocities (the cylinder spatters or flows laterally parallel to the target surface like a stream or fluid). Fig. 1 shows a scheme of the two deformation modes. In addition, White assumed a material transitional velocity $(\beta)$. If impact velocity is above $\beta$, the cylinders deforms as Mode II; if impact velocity is below $\beta$, the cylinders deforms as Mode I. For an elastic-perfect plastic material model with constant density, transitional velocity is given by Eq. (1), where $Y$ is the dynamic resistance of the material and $\rho$ is his density. Due the projectile is decelerating across the process, cylinder could suffer both deformation modes over the impact event.

$$
\beta^{2}=\frac{Y}{\rho}
$$

In this paper, an engineer/analytical model is approached to study the behavior of multipart cylinder impacting a rigid wall (See Fig. 2). The cylinder is composed of two materials: a hard one on front and a soft one at the rear part of the projectile. Materials are modeled as rigidperfect plastic with a constant density, using the concept of two modes of deformation and the transitional velocity developed by White [10].

\section{Preliminary concepts}

According to White [10], when a cylinder impacts a rigid surface with a velocity below transitional velocity, it deforms as Mode I (See Fig. 1). Then, equations govern the state of the cylinder can be obtain from the Taylor model [3]; where the continuity equation in the system (Eq. (2)), the mass and momentum equations of non-deformable part 


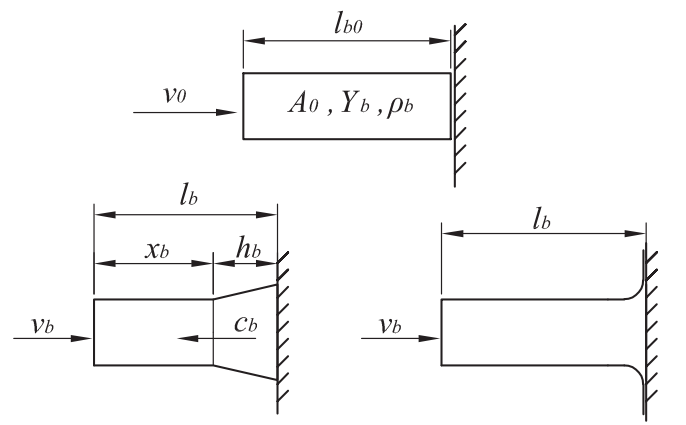

Fig. 1. Cylinder impacts a rigid wall at different velocities: below (left) and above (right) the transitional velocity, then cylinder can be deformed as Mode I or Mode II respectively.

(Eq. (3)), and the jump conditions for mass and momentum on the plastic-elastic interface (Eq. (4)) are specified.

$$
\begin{gathered}
l_{b}=x_{b}+h_{b} \quad \text { where } \quad \frac{d h_{b}}{d t}=c_{b} \\
\frac{d x_{b}}{d t}=-\left(v_{b}+c_{b}\right) \quad \text { and } \quad \rho_{b} x_{b} \frac{d v_{b}}{d t}=-Y_{b} \\
A_{0}\left(v_{b}-c_{b}\right)=A_{b} c_{b} \quad \text { and } \\
\rho_{b} A_{0}\left(v_{b}-c_{b}\right) v_{b}=Y_{b}\left(A_{b}-A_{0}\right)
\end{gathered}
$$

On the other hand, if the impact velocity is above transitional velocity, it deforms as Mode II (See Fig. 1). Then, equation govern the state of cylinder can be obtain from the Wilkins model [4], where the mass and momentum equations of the non-deformable part are given by Eq. (5).

$$
\frac{d l_{b}}{d t}=-v_{b} \quad \text { and } \quad \rho_{b} l_{b} \frac{d v_{b}}{d t}=-Y_{b}
$$

Assuming initial conditions $\left(v_{0}, l_{0}\right.$ and $\left.A_{0}\right)$ and material properties $\left(Y_{b}\right.$ and $\left.\rho_{b}\right)$; state equations can be numerically solved to obtain the deceleration and change of length of projectile.

\section{Model approach}

Projectile is composed by two cylinders impacting a rigid wall, as shown in Fig. 2. Cylinder " $a$ " can be deformed as Mode I or Mode II depending of his transitional velocity $\left(\beta_{a}\right)$. State equations can be achieved at same way to equations for a single cylinder (See Section 2. If $v_{a}>\beta_{a}$ (Mode II) conservation equations are given by Eq. (6).

$$
\frac{d l_{a}}{d t}=-v_{a} \quad \text { and } \quad \rho_{a} l_{a} \frac{d v_{a}}{d t}=-Y_{a}+Y_{b}
$$

On the other hand, if $v_{a}<\beta_{a}$ (Mode I), continuity and conservations conservation equations and jump conditions over the interface, are given by Eq. (7), Eq. (8) and Eq. (9) respectively.

$$
l_{a}=x_{a}+h_{a} \quad \text { where } \quad \frac{d h_{a}}{d t}=c_{a}
$$

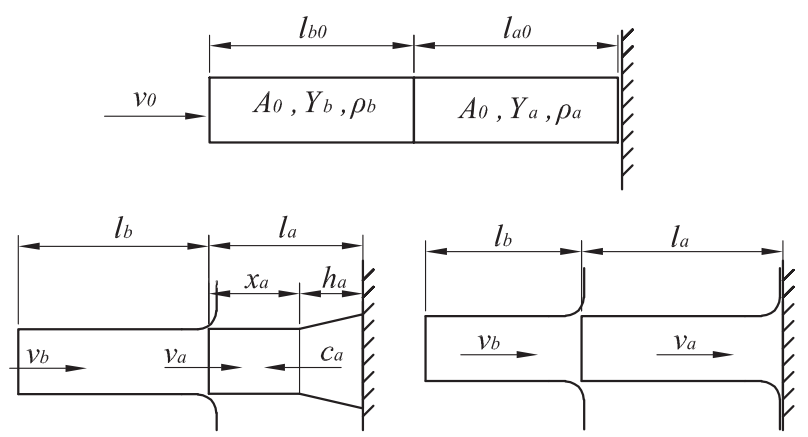

Fig. 2. Impact problem involving a multi-material cylinder against a rigid target: both cylinders in Mode II (right) and cylinder "a" in mode I and "b" in Mode II (left).

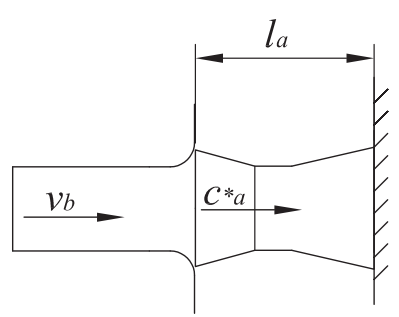

Fig. 3. Shrinkage of cylinder " $a$ " due the inertial forces of cylinder " $b$ ".

$$
\begin{gathered}
\frac{d x_{a}}{d t}=-\left(v_{a}+c_{a}\right) \quad \text { and } \quad \rho_{a} x_{a} \frac{d v_{a}}{d t}=-Y_{a}+Y_{b} \\
A_{0}\left(v_{a}-c_{a}\right)=A_{a} c_{a} \text { and } \\
\rho_{a} A_{0}\left(v_{a}-c_{a}\right) v_{a}=Y_{a}\left(A_{a}-A_{0}\right)
\end{gathered}
$$

It is assume that cylinder " $b$ " is always in contact with cylinder " $a$ ". Additionally, it is usual to use a strength material at front for ballistic applications (a hard nose); therefore, it is also assumed that $\beta_{a}>\beta_{b}$. Thus, cylinder " $b$ " is deformed in Mode II and its conservation equations are given by Eq. (10).

$$
\frac{d l_{b}}{d t}=\left(v_{a}-v_{b}\right) \quad \text { and } \quad \rho_{b} l_{b} \frac{d v_{b}}{d t}=-Y_{b}
$$

When cylinder " $a$ " stops, the interface acts as a rigid wall for cylinder " $b$ ". However, rear part of " $a$ " suffers an additional shrinkage due the inertial forces of " $b$ " (see Fig. 3). Then, mass and momentum conservation equation to the real portion of " $a$ " can be proposed as:

$$
\begin{gathered}
\frac{d l_{a}}{d t}=c *_{a} \\
A_{a}\left(c *_{a}-v_{b}\right)=A_{0} c *_{a} \quad \text { and } \\
\rho_{a} A_{a}\left(v_{b}-c *_{a}\right) v_{b}=Y_{a}\left(A_{0}-A_{a}\right)
\end{gathered}
$$

Finally, when " $a$ " shrinkage stops, cylinder " $b$ " behaviour can be modeled as a single cylinder according with its velocity (Equations (2), (3), (4) or Equations (5)). 

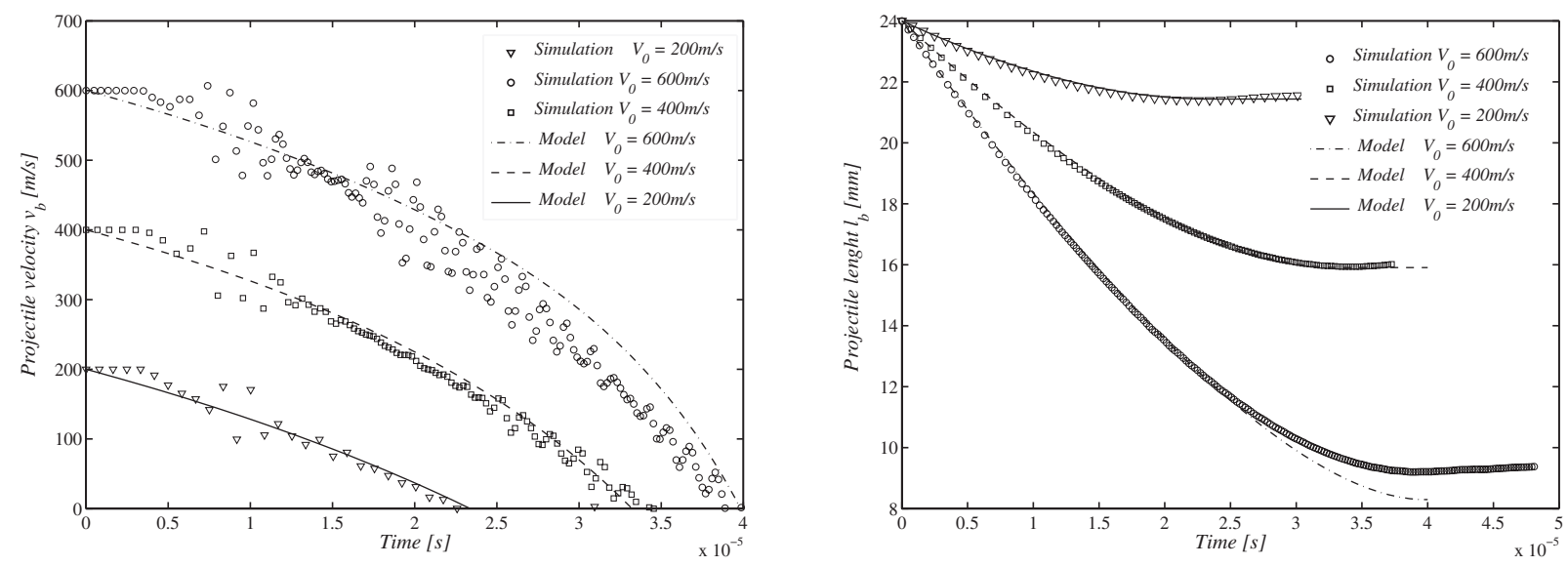

Fig. 4. Impact of a single cylinder against a rigid target: (a) Comparison of cylinder velocity change, and (b) Comparison of cylinder length change between analytical model and simulation at different initial velocities.

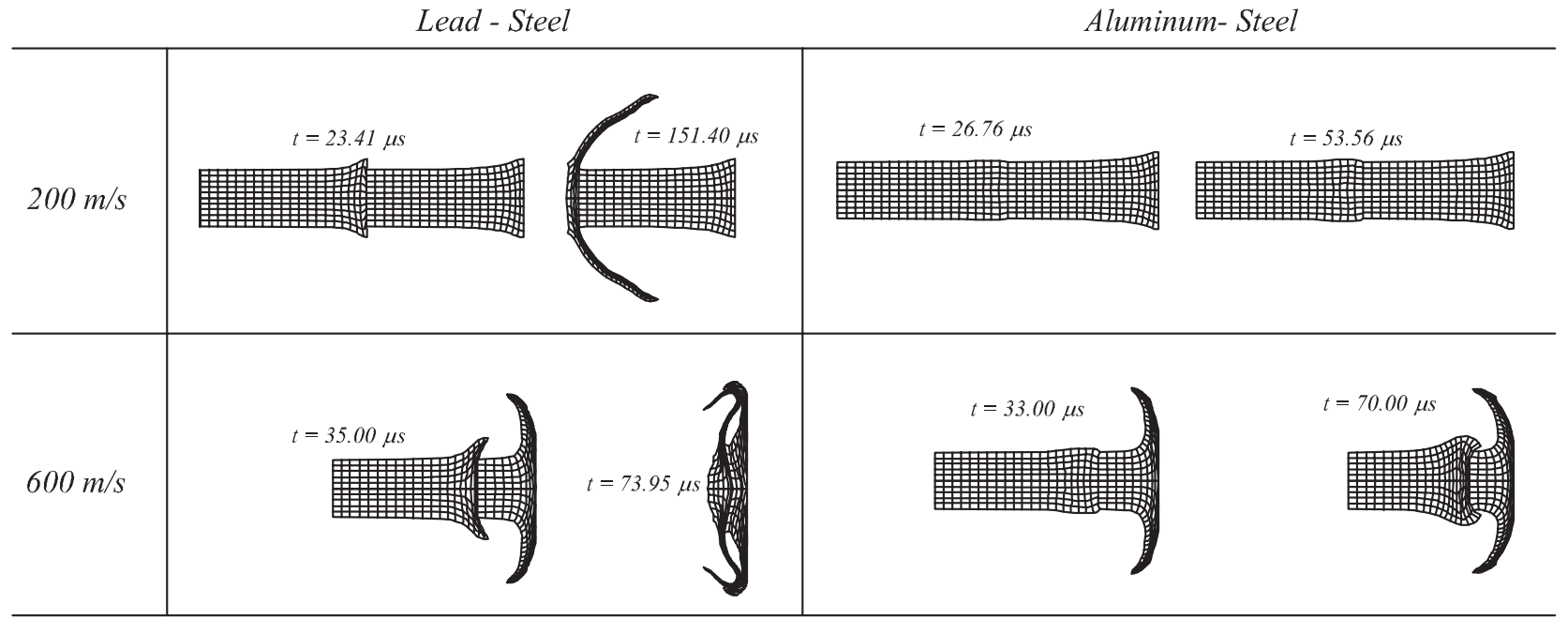

Fig. 5. Simulation impact sequences of cylinders at $V_{0}=200 \mathrm{~m} / \mathrm{s}$ and $V_{0}=600 \mathrm{~m} / \mathrm{s}$ for the two configurations studied. It is shown the state at intermediate and final arrest time.

Table 1. Multi-material cylinder comfigurations for checking the model.

\begin{tabular}{ll}
\hline Cylinder "b" & Cylinder "a" \\
\hline Lead & Steel \\
Aluminum & Steel \\
\hline
\end{tabular}

\section{Methodology}

The model was verified using a multi-material cylinder combining two different metals, as can be seen in Table 1. Each cylinder is $7.62 \mathrm{~mm}$ in diameter and $24 \mathrm{~mm}$ long. Materials used for modelling are shown in Table 2. Transitional velocity $\beta$ was calculated for each material according to Eq. (1).

Differential equation systems were programmed and solved using a Newton-Rapston numerical method with 1E-9 time step. Additionally, computational simulations were made using AUTODYN 2D axisymmetric simulations with an $0.25 \mathrm{~mm}$ element size. Results were compared and evaluated for corrections.
Table 2. Materials used for verifing the model and their properties.

\begin{tabular}{llll}
\hline & $\begin{array}{l}\text { Dynamical } \\
\text { Resitance } \\
\text { Material }\end{array}$ & $\begin{array}{l}\text { Density } \\
\rho[\mathrm{MPa}]\end{array}$ & $\begin{array}{l}\text { Transitional } \\
\text { velocity } \\
{\left[\mathrm{kg} / \mathrm{m}^{3}\right]}\end{array}$ \\
$\beta[\mathrm{m} / \mathrm{s}]$ \\
\hline Lead & 68 & 11340 & 77 \\
Aluminum & 350 & 2700 & 360 \\
Steell & 1200 & 7800 & 392 \\
\hline
\end{tabular}

\section{Results and discussion}

Firstly, single cylinder impacts were modeled in order to verify and to calibrate material properties using equations (2), (3), (4) or equation (5). Results were compared with simulation for different velocities. As such, comparison was performed for a single cylinder of steel at different velocities (Fig. 4).

According to simulation sequence in Fig. 5, cylinder "b" of lead deformed as Mode II at the two cases modeled because its $\beta_{\text {lead }}$ is lower that the impact velocities; 

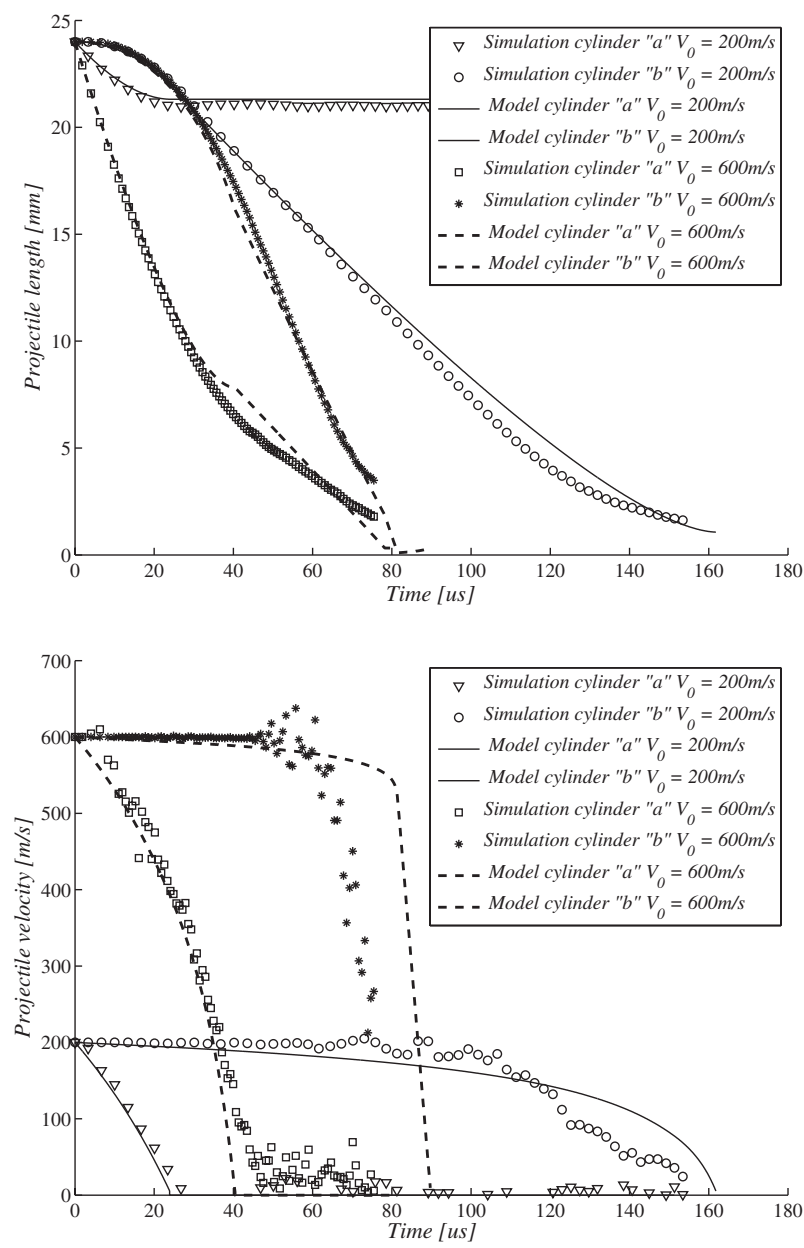

Fig. 6. Lead-Steel cylinder: (a) Comparison of the cylinder length change between analytical model and simulation at different initial velocities and, (b) Comparison of the cylinder velocty change between analytical model and simulation at different initial velocities.

whereas, cylinder "b" of aluminum deformed as Mode II for $v_{0}=600 \mathrm{~m} / \mathrm{s}$ and as Mode I for $v_{0}=200 \mathrm{~m} / \mathrm{s}$ due its higher $\beta_{\text {aluminum. }}$. Cylinder "a" of steel behaves equal to aluminum cylinder due their similar transitional velocities (See Table. 2). Note that in Mode I, cylinders only change its shape as a mushroom, but it did not spatter on the rigid wall, as in Mode II. Figures 6(a) and 7(c) compare the length change and velocities of each cylinder for the two configuration. It can be seen good agreement between simulation and analytical model, although it is lower at high impact velocities. Some reasons for the lower agreement at high velocities are the assumption of a constant dynamical resistance because it has been proven that it could be very sensitive at different velocities, and even more for aluminum [4]. Additionally, if velocity is high enough, cylinder " $b$ " penetrates cylinder "a"; thus, model could lost its accuracy. According to Tate [10,11], a projectile stops to penetrate a target when its velocity is lower than a critical velocity $\left(v_{\text {crit }}\right)$. This velocity can be calculated making zero the velocity of penetration (U) in the hydrodynamic equation Eq. (13); thus, $v_{\text {crit }}$ can be expressed by Eq. (14) (Subscripts were changed
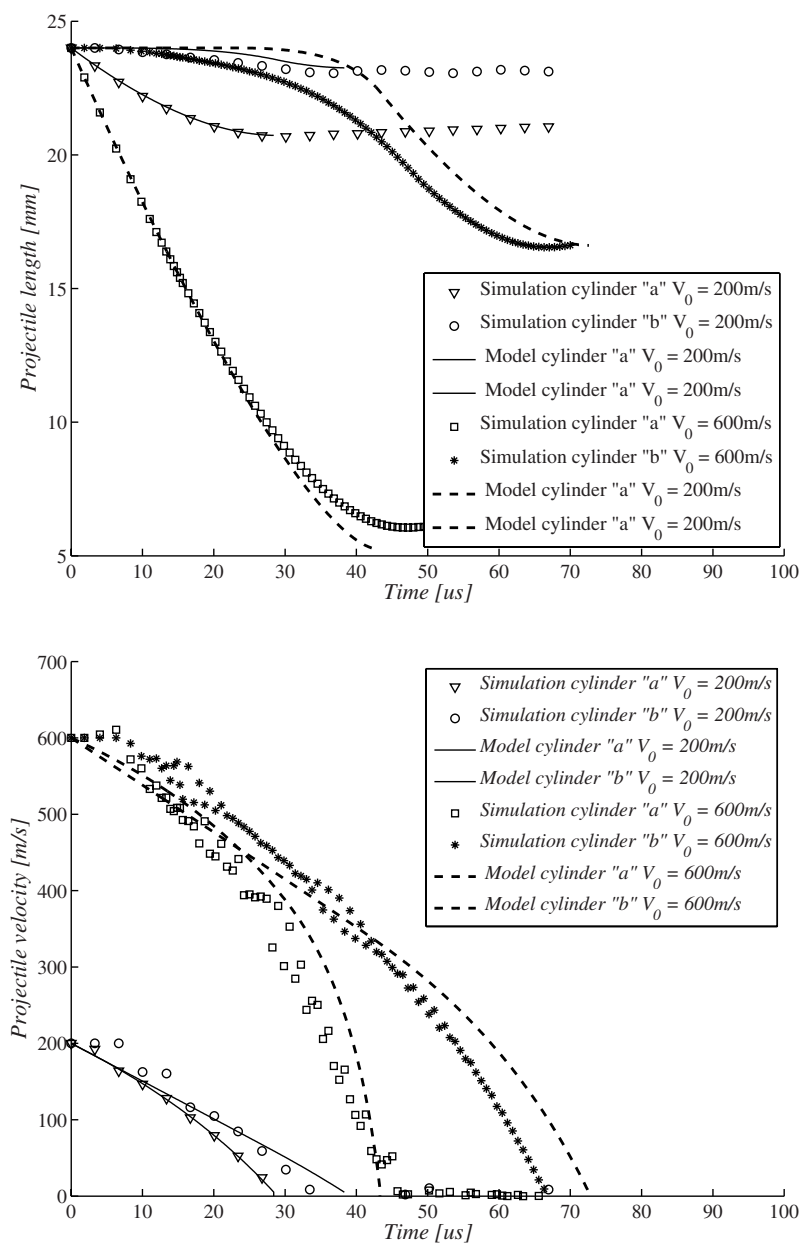

Fig. 7. Aluminium-Steel cylinder: (a) Comparison of Cylinder length change between analytical model and simulation at different initial velocities and, (b) Comparison of Cylinder velocty between analytical model and simulation at different initial velocities.

corresponding to current nomenclature).

$$
\begin{aligned}
R_{t}+\frac{1}{2} \rho_{t} U^{2} & ==Y_{p}+\frac{1}{2} \rho_{p}(v-U)^{2} \\
v_{\text {crit }} & =\frac{2\left(Y_{a}-Y_{b}\right)}{\rho_{b}}
\end{aligned}
$$

Then, model shows good agreement at velocities below $v_{\text {crit }}$, which it is calculated for materials involved in the multi-material cylinder.

\section{Conclusion}

An engineering model is proposed for modeling the behavior of a unique cylinder composed of two different metals cylinders when impacts on a rigid surface. It was found good agreement between model and simulation results; moreover if the impact velocities are below the critical velocity deduced by Tate theory. 


\section{Acknowledgements}

The authors acknowledge the financial support to this research given by Universidad de los Andes.

\section{References}

1. P. J. Hogg, Proceedings of Composite Processing, (2003)

2. R. H. Berlin et al., The Journal of trauma, 28,1 (1988) p.75

3. G. Taylor, Proceedings of the Royal Society of London. Series A. Mathematical and Physical Sciences, 194,1038 (1948). p.289

4. M. L. Wilkins y M. W. Guinan, Journal of Applied Physics, 44,3 (1973). p.1200
5. E. H. Lee y S. J. Tupper, J. appl. Mech., 21,1 (1954). p.63

6. J. B. Hawkyard, International Journal of Mechanical Sciences, 11,3 (1969). p.325

7. S. E. Jones et al., Journal of applied physics, 61,2 (1987). p.499

8. S. E. Jones et al., NASA STI/Recon Technical Report, 96,1038 (1948). p.17955

9. S. E. Jones et al., International journal of impact engineering, 21,1-2 (1998). p.1

10. M. P. White, Journal of applied mechanics, 51,1 (1984). p.102

11. A. Tate, Journal of the Mechanics and Physics of Solids, 15,6 (1967). p.387

12. A. Tate, Journal of the Mechanics and Physics of Solids, 17,3 (1969). p.141 